\title{
Intrasurgical Site Route of Administration
}

National Cancer Institute

\section{Source}

National Cancer Institute. Intrasurgical Site Route of Administration. NCI Thesaurus.

Code C142365.

Administration within the site of surgery. 